Diabetische Neuropathie

\section{Was empfehlen die Leitlinien?}

Die diabetische Neuropathie ist neben der Nephropathie und der Retinopathie eine der häufigsten Komplikationen des Diabetes mellitus. Die Praxisleitlinie der Deutschen Diabetes-Gesellschaft (DDG) empfiehlt neben Basismaßnahmen eine Reihe verschiedener Substanzen, die bei starken Schmerzen auch in Kombination eingesetzt werden sollten.

- Die distale Neuropathie gehört zu den häufigsten Komplikationen bei Diabetikern: Jeder vierte Typ-1-Diabetiker und jeder zweite Typ-2-Diabetiker ist betroffen. Eine schmerzhafte Polyneuropathie entwickeln nur ca. 5\% der Typ-1-, aber ca. $25 \%$ der Typ-2-Diabetiker. „Bei den diabetischen Neuropathien muss man die distal-symmetrische Polyneuropathie und die autonome Neuropathie unterscheiden“, erklärte Prof. Jochen Seufert, Freiburg. Dazu kommen mit Diabetes assoziierte Neuropathien wie die fokale und multifokale Neuropathie, die thorakale Radikulopathie, Hirnnervenparesen und Nerven-Engpass-Syndrome.

Neuropathische Schmerzen beeinträchtigen die Lebensqualität erheblich, zumal sie mit weiteren Symptomen wie Schlaf- und Angststörungen, Depression und Gewichtsverlust assoziiert sind.

Die wichtigste präventive Maßnahme ist die Optimierung der Diabeteseinstel-

\section{Empfehlungen der Deutschen Gesellschaft für Neurologie}

\section{Leitsätze für die medikamentöse Therapie}

- Das wirksamste Medikament muss für jeden einzelnen Patienten individuell unter Berücksichtigung seiner Beschwerden sowie der Nebenwirkungen und Kontraindikationen gefunden werden.

- Jeder Patient benötigt eine individuelle Dosierung in Abhängigkeit von Wirkung und Nebenwirkungen, d. h. eine sorgfältige Titration.

- Die Wirkungslosigkeit eines Medikaments sollte erst nach zwei bis vier Wochen unter ausreichender Dosierung beurteilt werden.

- Einzeldosen und Applikationsintervalle müssen je nach Pharmakokinetik und Interaktionsprofil bemessen werden. lung. Auch die Vermeidung weiterer Risikofaktoren wie Alkohol, Rauchen und Übergewicht gehört zur Prävention.

\section{Die Qual der Wahl}

Für die Therapie der diabetischen Polyneuropathie steht ein breites Spektrum verschiedener Substanzen zur Verfügung, erklärte Prof. Jan Ziegler, Düsselsam und daher obsolet. Dagegen können Paracetamol oder Metamizol bei intermittierenden leichten Schmerzen kurzfristig eingesetzt werden. Bei chronischen Schmerzen empfiehlt die Praxisleitlinie der DDG als Mittel der ersten Wahl Antidepressiva wie Duloxetin, Amitriptylin, Clomipramin und Imipramin. Auch der Kalziumkanalmodulator Pregabalin wird empfohlen. „Es moduliert die Aktivität überaktivierter Schmerzfasern und trägt somit zu einer signifikanten Schmerzreduktion bei", so Seufert.

- Bei gleicher analgetischer Wirksamkeit sollen Medikamente bevorzugt werden, deren Risiko für kardiovaskuläre, hepatische, renale und metabolische Nebenwirkungen am niedrigsten ist.

- Kombinationspräparate mit Koffein, Benzodiazepinen oder Muskelrelaxanzien sind nicht indiziert und bergen die Gefahr von Missbrauch und Abhängigkeit.

- Realistische Therapieziele sind eine Schmerzreduktion um 30-50\%, eine Verbesserung der Schlaf- und Lebensqualität, die Erhaltung der sozialen Aktivität und der Arbeitsfähigkeit. dorf. NSAR und Coxibe seien nicht wirk-

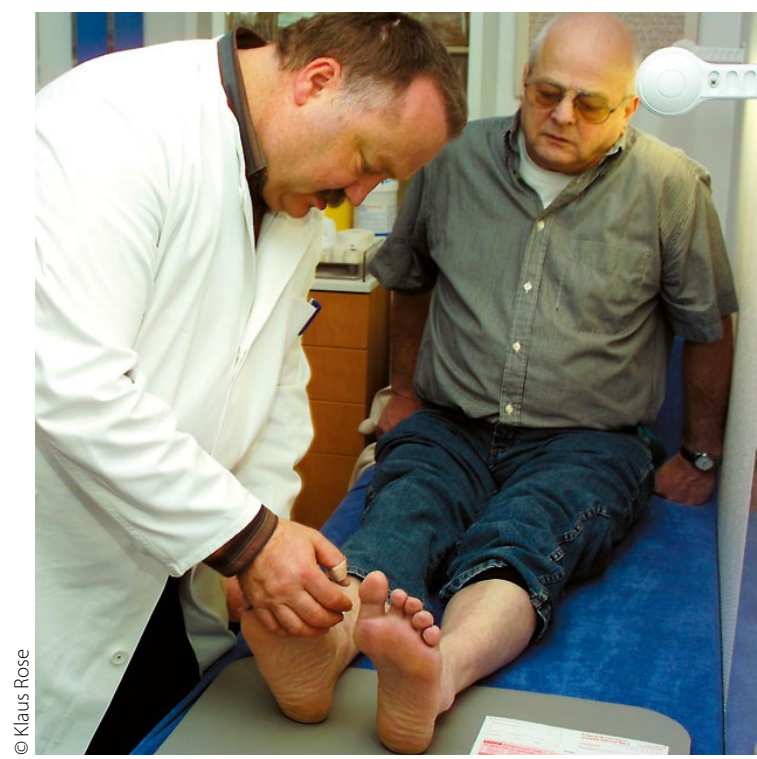

Jeder vierte Typ-2-Diabetiker entwickelt eine schmerzhafte Polyneuropathie.

Als Medikamente der zweiten Wahl werden Opioide wie Tramadol und Oxycodon, der Kalziumkanalmodulator Gabapentin und alpha-Liponsäure propagiert. Letztere sollte als intravenöse Infusion über drei Wochen gegeben werden. In der Nationalen Versorgungsleitlinie wird alpha-Liponsäure allerdings nicht empfohlen. Mittel der dritten Wahl sind der Natriumkanalblocker Carbamazepin und Capsaicin lokal.

Bei ausgeprägter Symptomatik empfiehlt sich eine Kombinationstherapie mit einem Opioid.

\section{Nicht medikamentöse Maßnahmen}

Einen hohen Stellenwert haben auch nicht pharmakologische Ansätze wie die Elektrotherapie als Nerven- und Muskelstimulation, die Physiotherapie und die Verhaltenstherapie. „Die Schmerzen können auch durch gezielte Ablenkung und eine Umlenkung der Aufmerksamkeit weg von belastenden Gedanken hin $\mathrm{zu}$ angenehmen Dingen, Pflegen sozialer Kontakte, körperliche Aktivität und sinnvolle Alltagsgestaltung besser bewältigt werden“, so Seufert.

Dr. med. Peter Stiefelhagen =

- Quelle: 46. Jahrestagung der Deutschen Diabetes-Gesellschaft am 1.6.2011 in Leipzig.

- Die neue Nationale Versorgungsleitlinie zur Diabetischen Neuropathie befindet sich noch in Überarbeitung: http://www.versorgungsleitlinien.de/themen/diabetes2/dm2_neuro 